\title{
BIENES DE RANGO ELEVADO EN EL COMERCIO AL POR MENOR; LUGARES CENTRALES EQUIPADOS EN LA REGION DE MURCIA
}

\author{
P O R
}

JOSE MARIA SERRANO MARTINEZ

Doctor en Geografía. Profesor del Departamento de Geografía General. Universidad de Murcia

\section{Sumario}

1. BIENES DE RANGO ELEVADO

2. PROCEDIMIENTO SEGUIDO

3. FUENTES

4. ANALISIS DE LOS BIENES DE RANGO ELEVADO

4.1. Tipología de los bienes

4.2. Total de unidades funcionales

4.3. Variedad funcional

4.4. Indice de Centralidad

4,5. Comparación de las distintas jerarqutas

5. CONCLUSIONES

NOTAS

\section{BIENES DE RANGO ELEVADO}

En el estudio más amplio por nosotros elaborado ${ }^{1}$, uno de los aspectos que estudiábamos era el comercio en general dentro del equipamiento funcional y terciario de los Lugares Centrales (en adelante L.C.). No es necesario insistir en la gran importancia que posee el comercio dentro del conjunto de los servicios ${ }^{2}:$ se trata de una realidad tan presente que no merece la pena insistir sobre ello.

Las ciudades tradicionalmente han venido desempeñando un papel de centros de mercado, sea como lugares de intercambio ${ }^{3}$ o ya en la actualidad, dentro de sociedades consumistas, como centros suministradores de bienes al que se desplazan los vecinos de

1 SERRANO MARTINEZ, J. M. ${ }^{\mathbf{a}}$ : La Red Urbana de Murcia. 6 volúmenes. Constituye la Tesis Doctoral del autor, leída en la Universidad de Murcia el 21 de enero de 1983.

2 Desde los estudios tradicionales de Christaller, siempre se ha dedicado una especial atención a conocer la movilidad geográfica originada por el comercio.

3 En este sentido como pionero de los estudios de Geografía sobre los centros de mercado en España, cabe citar la obra de CASAS TORRES, J. Ml. y ABASCAL GARAYOA, A.: Mercados geográficos y ferias en Navarra. Zaragoza, 1948. 
cada L.C. situados dentro de su área funcional para aprovisionarse de ellos. De ahí, se deriva la necesidad de conocer el equipamiento funcional en estos bienes para determinar a través de ellos sus espacios de influencia, bien simplemente con una misión especulativa o como medio de otras aplicaciones prácticas.

El comercio al por menor supone en esencia para su realización una ácción volitiva de carácter individual. Posee esencialmente dos connotaciones elementales:

- Generalización

- Cotidianidad

Estos rasgos lo separan de otras funciones terciarias y del mismo comercio al por mayor. No obstante, si abarcamos en conjunto toda la amplia gama de bienes comprendidos en la denominación de actividades comerciales se corre el riesgo de generalizar demasiado el número de L.C. que expiden tales bienes, derivándose de ello una imprecisión en lo que se refiere a su capacidad para determinar áreas funcionales.

Nuestro propósito consiste en conocer, específicamente, sólo aquellas funciones comerciales, que poseyendo un rango más elevado, es decir una especialización selectiva en su oferta, exigen un área de mercado mayor para subsistir ${ }^{4}$. Como consecuencia se localizan dichos bienes en aquellas ciudades, bien de mayor tamaño demográfico o que por especiales circunstancias extienden su área de influencia sobre otros espacios y los L.C. comprendidos en ellos.

Cuando analizamos el comercio al por menor en la Región de Murcia, encontramos que existen ciertos bienes presentes en casi en todos los L.C. 5 incluso en los de menor tamaño demográfico.

Es necesario tener en cuenta que, por causas de tipo histórico, nuestra Región posee una división con un muy reducido número de términos municipales en comparación con la casi totalidad de todo el territorio nacional. Ello no obsta para que el poblamiento comprenda multitud de asentamientos dispersos o concentrados sin incluir en tal calificación administrativa. Pero ciñéndonos a la categoría de municipios, abundan los que cabe considerar de un tamaño demográfico medio ${ }^{6}$ e incluso grande.

De acuerdo con el criterio matemático empleado en la determinación del rango de los bienes, especificado en la correspondiente nota ${ }^{7}$, sólo vamos a considerar de rango elevado aquellos que superan la magnitud de 0,60. Eso significa que están presentes en $18 \mathrm{~L}$.C. del total de la Región o en un número inferior a esa cifra. Lo cual determina a priori que toda persona para acceder a su consumo tendrá que desplazarse desde su lugar de origen a uno de esos puntos donde se expenden tales mercancias. De ahí que dichos centros expendedores se conviertan, automáticamente, en L.C. con una preeminencia notable sobre el conjunto de los asentamientos de población del espacio regional.

4 Véase por ejemplo lo que escribe B.J.L. BERRY sobre ello en su obra Geografía de los centros de Mercado y distribución al por menor. Barcelona, 1971, Ed. Vicens Vives, págs. 95-114.

5 Nos referimos obviamente a aquellos bienes de rango más reducido, tales como venta de comestibles, pescaderías, droguerías, etc.

6 Ya el número total de municipios en la Región es reducido, sólo 44, de ahí en gran parte la causa de ese fenómeno.

7 Para la determinación del rango de los bienes empleamos la fórmula que cita PRECEDO LEDO, A. J.: La Red Urbana de Navarra. Pamploma, 1976, págs. 128 y ss.

$$
\mathrm{ri}=1-\frac{\mathrm{Fi}}{\mathrm{N}}
$$

ri Rango de los bienes centrales (De 0 a 1 ).

Fi Número de Lugares Centrales que poseen el bien i.

N Número total de Lugares Centrales. 
Pero el problema incluso se acentúa; ya que en el conjunto de los bienes seleccionados se confirma un descenso de los lugares equipados, en beneficio de un número muy reducido de ellos.

\section{PROCEDIMIENTO SEGUIDO}

A partir del rango elevado de estos bienes, dentro del conjunto general del equipamiento funcional del comercio, realizamos un estudio detenido de:

- Total de unidades funcionales y su distribución (T.U.F.).

- Análisis de la variedad funcional de cada L.C. (V.F.).

- Determinación del Indice de Centralidad de los L.C. y su estudio diferencial (I.C.).

- Comparación entre las anteriores jerarquías y el tamaño degráfico de los L.C.

- Representación cartográfica, mediante gráficos y tablas que contienen datos de las magnitudes analizadas.

- Elaboración de unas conclusiones.

El procedimiento utilizado consiste pues, en combinar un método analítico, descriptivo, de sintesis y su cuantificación; donde la cartografía nos sirve, especialmente, para mostrar el conjunto de los datos analizados y las conclusiones que de ellos se obtienen.

\section{FUENTES}

Los datos que empleamos proceden sobre todo de las licencias fiscales (Delegaciones de Hacienda de Murcia y Cartagena, año 1980 septiembre, y del Censo de población I.N. de Estadística 1 de marzo de 1981).

Ambas fuentes son muy utilizadas en trabajos geográficos, y pensamos no necesitan ni justificación ni explicación. Quizás sólo sea conveniente recordar que las licencias fiscales no corresponden siempre con el número de establecimientos existentes, en especial en estos bienes de rango elevado. Ello se debe a que ciertos negocios si bien no poseen la licencia específica para vender los bienes citados, es frecuente que algunos de estos, ocasionalmente o de forma parcial, se encuentren entre sus mercancias.

Creemos sin embargo que estos casos no producen graves distorsiones en nuestro estudio, ya que las licencias fiscales aparecen, precisamente, cuando el volumen de negocio realizado alcanza una cota considerable, hasta tal punto que su propietario considere necesario "darse de alta" en esa licencia, para evitar cualquier problema que pudiera derivarse de su omisión. Por tanto la existencia de la licencia fiscal, supone proprocionalmente un alto grado de especialidad en la comercialización del producto y de venta de la mercancía.

Como complemento de lo anterior, y en lo que se refiere a productos muy especializados, se consultaron parcialmente los datos que los ayuntamientos respectivos poseían. 


\section{ANALISIS DE LOS BIENES DE RANGGO ELEVADO}

\subsection{Tipología de los bienes}

Como puede verse en la tabla número 1 es muy variada la gama de bienes que se han analizado. Resultan comprendidos, desde los que indican un grado de especialización del comercio más elemental (caso de la venta de géneros de punto, alfombras o libros nuevos) hasta otros situados en un nivel medio de especialización (ópticas, máquinas de escribir y calcular; venta de anuncios); o bien aquellos que indudablamente, señalan con su rango elevado la existencia de un área funcional muy amplia, alcanzando incluso a toda la Región, y aún sobrepasan ésta: tales como la venta de audífonos, grandes almacenes, material de ortodoncia, antigüedades, etc.

Otro rasgo que puede resaltarse es la variedad general de los bienes existentes en este sentido. La gama de servicios que ofrecen es amplia y cubre prácticamente un extenso campo de posibles necesidades.

Descuella también el hecho peculiar de la distribución del conjunto de las funciones en los correspondientes núcleos urbanos. De los 44 bienes analizados, solamente seis están presentes en más de diez ciudades. De estas, sólo dos superan la cifra de quince. El número cinco, referido a L.C. dotados, es uno de los más repetidos. Quizás lo que se echa de menos es la inexistencia de una serie de bienes que estén presentes en las tres ciudades mayores, como sucede con otros muchos servicios terciarios, no comerciales, y que sólo ocurre en este caso en Murcia y Cartagena. 
sə[หuo!̣uny sopepiun IR7o.

eุo.nก $\mathrm{x}$

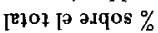
[eๆo) [ə әxq̣os\%

Rนวลิвา.IRว นว [e]o] ja axqos\% sорвาор

вฺุว Х

uog!u

eurzoL

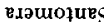

Jə̣̂R [ UES

อเว.In以

EโnW

S әр вuп़oW

uọjreze W

ต.IO 1

EII!Uu!

Ezว!?

นเรวบวว

อนวอีอนมอว

eocatej

euวपว.5

हueपIV

eโtนrequeว

se[!na

ue,req

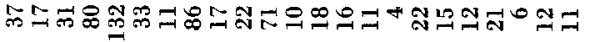

⿻ㅝㄱำㄱำำำ

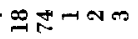

$m+a+\pi$ man

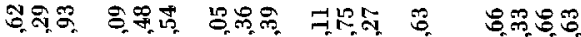

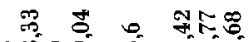

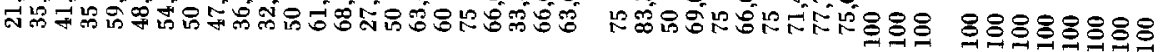

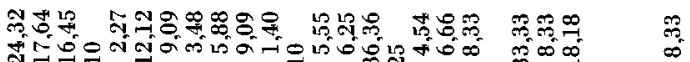

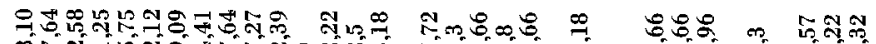

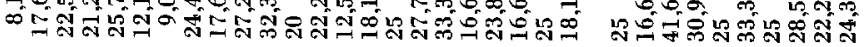

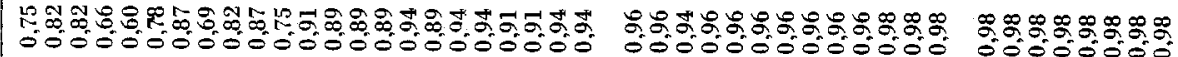

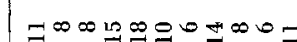

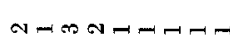

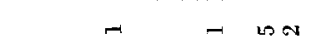

N $\rightarrow-\infty \rightarrow$

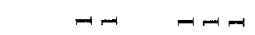

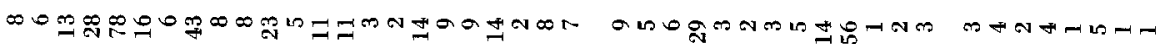

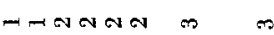
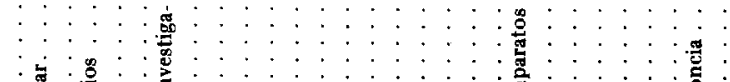

$\therefore$ :

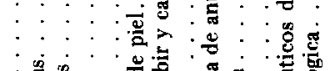

递实

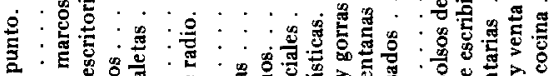

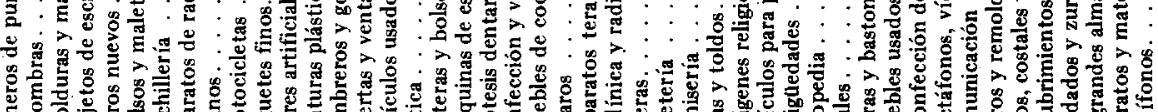




\subsection{Total de unidades funcionales}

Dentro del esquema general a seguir en su estudio, un punto importante, en el que nos detendremos es el análisis del T.U.F. Ascienden a 904. Ciertamente su distribución no sigue, como pudiera pensarse un orden proporcional con el tamaño demográfico. En el cuadro que viene a continuación se especifica esto:

\section{Cuadro número 1}

Total Unidades Funcionales

\begin{tabular}{|c|c|c|c|}
\hline A & $\mathrm{B}$ & $\mathrm{C}$ & D \\
\hline 1 & Murcia ........... & 491 & 54,34 \\
\hline 2 & 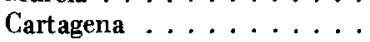 & 205 & 22,42 \\
\hline 3 & Lorca . . . . . . . . . & 53 & 5,86 \\
\hline 4 & Cieza . . . . . . . & 22 & 2,43 \\
\hline 5 & Caravaca . . . . . . & 19 & 2,10 \\
\hline 6 & Aguilas . . . . . . . . . & 18 & 1,99 \\
\hline 7 & Molina de Segura . . . . . . & 16 & 1,76 \\
\hline 8 & 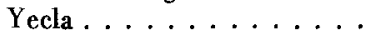 & 15 & 1,65 \\
\hline 9 & Alcantarilla . . . . . . & 10 & 1,10 \\
\hline 10 & La Unión. . . . . . . . . & 9 & 0,99 \\
\hline 11 & 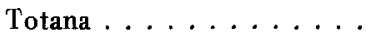 & 8 & 0,88 \\
\hline 12 & Jumilla . . . . . . . . . & 7 & 0,77 \\
\hline 13 & Alhama. . . . . . . . . & 7 & 0,77 \\
\hline 14 & 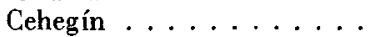 & 6 & 0,66 \\
\hline 15 & San Javier $\ldots \ldots \ldots$ & 5 & 0,55 \\
\hline 16 & Mazarrón. . . . . . . & 5 & 0,55 \\
\hline 17 & $\ldots \ldots \ldots$ & 4 & 0,44 \\
\hline 18 & $\ldots \ldots \ldots$ & 2 & 0,22 \\
\hline 19 & $\ldots \ldots$ & 1 & 0,11 \\
\hline \multirow[t]{2}{*}{20} & Santomera . . . . . . . & 1 & 0,11 \\
\hline & & 904 & 99,9 \\
\hline
\end{tabular}

A Número de orden en la jerarquía.

B Nombre de la ciudad.

C Total de unidades funcionales.

D Porcentaje sobre el total.

Observamos lo siguiente:

- Predomina dentro de los L.C. más dotados, Murcia. El número de unidades funcionales que posee resulta proporcionalmente superior al que corresponde a su tamaño demográfico. Comprende ella sola el 54,34 \% del total, es decir 491 unidades funcionales.

- Le sigue también con un elevado grado de importancia Cartagena; 205 U.F. y el $22,42 \%$. Aunque como cabe observar, su equipamiento es proporcional y absolutamente más reducido.

- A partir de este L.C., la dotación del T.U.F. sufre una baja muy considerable, ya que Lorca $\left(3 .^{\circ}\right.$ lugar en la jerarquía citada) posee un conjunto muy reducido de unidades.

- Es a partir de esta ciudad cuando se hace más notable el descenso progresivo de Ios valores alcanzados, correspondientes al resto de los L.C., que presentan cifras muy reducidas. Después de Yecla ( $8 .^{\circ}$ lugar en la jerarquía), el equipamiento se reduce tanto que prácticamente se convierte en testimonial. Pensamos que en los lugares postreros, con su reducida dotación de U.F., apenas si cumplen el cometido principal, motivo de este estudio.

$$
-100-
$$


Comparando las jerarquías correspondientes con la resultante del tamaño demográfico y las relativas a la dotación funcional total, las alteraciones que se producen en ambas son notables. Más adelante se analizarán con detenimiento.

El umbral referente al tamaño demográfico-equipamiento funcional lo ocupa Santomera (7.346); aunque su presencia más bien parece testimonial ya que sólo posee una U. F.

Por otro lado es significativo, y entra dentro de lo normal, que de todas las funciones utilizadas, su gran mayoría, al contemplarlas singularmente, sólo alcanzan unos valores absolutos reducidos. En más del $95 \%$ de los casos, el T.U.F. con que aparecen dotados los L.C., dentro de cada función, es inferior de 6 unidades. Las excepciones más llamativas al respecto son pocas. Citamos, a título de ejemplo, las muy significativas:

- V. m. libros nuevos, Murcia posee 78 U.F. y Cartagena 34.

- V. m. objetos de escritorio, Murcia 28 U.F., Cartagena 17.

- V. m. juguetes finos, Murcia 23 U.F., Cartagena 23.

- V.m. optica, Murcia 14 U.F.y Cartagena 5, etc.

Todo ello viene a confirmar el carácter selectivo de este comercio; hecho que apuntábamos al principio. Esto se manifiesta de una forma doble:

a) Sólo unos pocos L.C., generalmente los de mayor tamaño demográfico aparecen equipados.

b) En ellos, la dotación de U.F. es casi siempre muy reducida.

Esta U.F. posee una amplia área funcional y un umbral de demanda muy elevado. Observando la tabla número $\bar{l}$, en la parte de la derecha, donde se expresa el porcentaje de las U,F: y su correspondiente distribución entre los 3 principales L.C., se alcanzan especialmente entre Murcia y Cartagena, valores muy por encima de los que corresponden a su tamaño demográfico, lo que evidencia su sobreequipamiento comercial en tales bienes.

Se confirma cuanto ha sido dicho, con lo expuesto en las figuras 1 y 2 donde quedan expresados los desplazamientos seguidos para comprar dos de los bienes incluidos en la relación estudiada ${ }^{8}$. Nos referimos a la compra de libros nuevos y al servicio de óptica. En ambas figuras descuellan ampliamente el papel nodal que ejerce la capital de la Región ya que centraliza flujos procedentes de casi todos los L.C. existentes. Escasean por otra parte, los L.C. activos; incluso estos, reciben unos flujos muy débiles, salvo el caso citado de Murcia-ciudad.

A fin de sintetizar lo expuesto, la figura número 3 representa, según la fórmula indicada en su pie, la distribución de las U.F. en los correspondientes lugares dotados; la número 4 , recoge dichos valores mediante un diagrama en barras.

\subsection{Variedad funcional}

El total de funciones analizadas son 44. Solamente Murcia-ciudad posée una dotación funcional que incluye todas. El resto sólo mantiene un equipamiento incompleto. Así Cartagena, segundo lugar en la jerarquía, posée una dotación restringida a 22 funcio-

8 Esas figuras son la representación cartográfica, fruto de una encuesta más amplia realizada en febrero-marzo de 1982, para determinar las áreas funcionales de la Región, sobre un número seleccionado de personas, en todos los municipios.

$$
-101-
$$




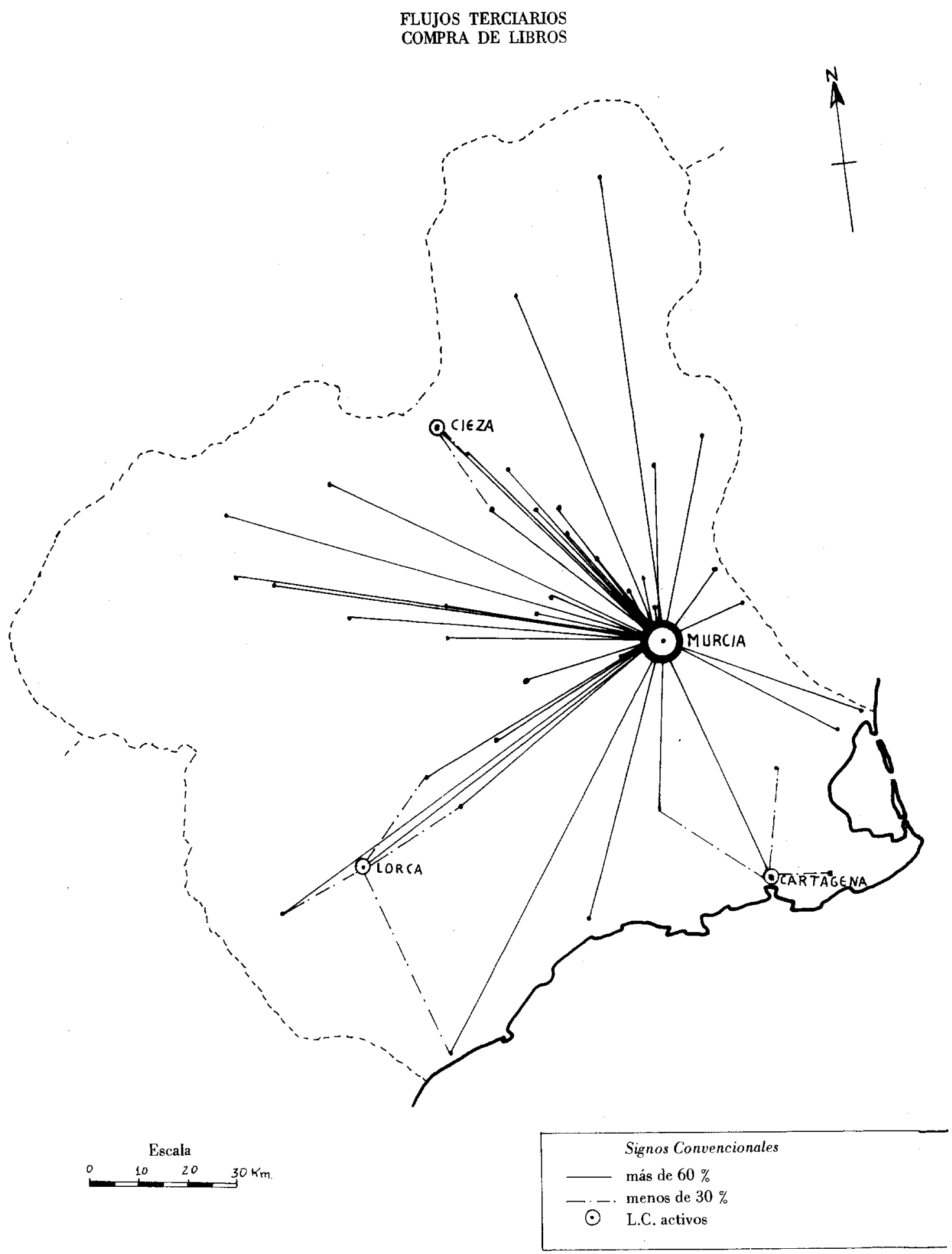

Figura núm. 1 
FLUJOS TERCIARIOS

SERVICIO DE OPTICA
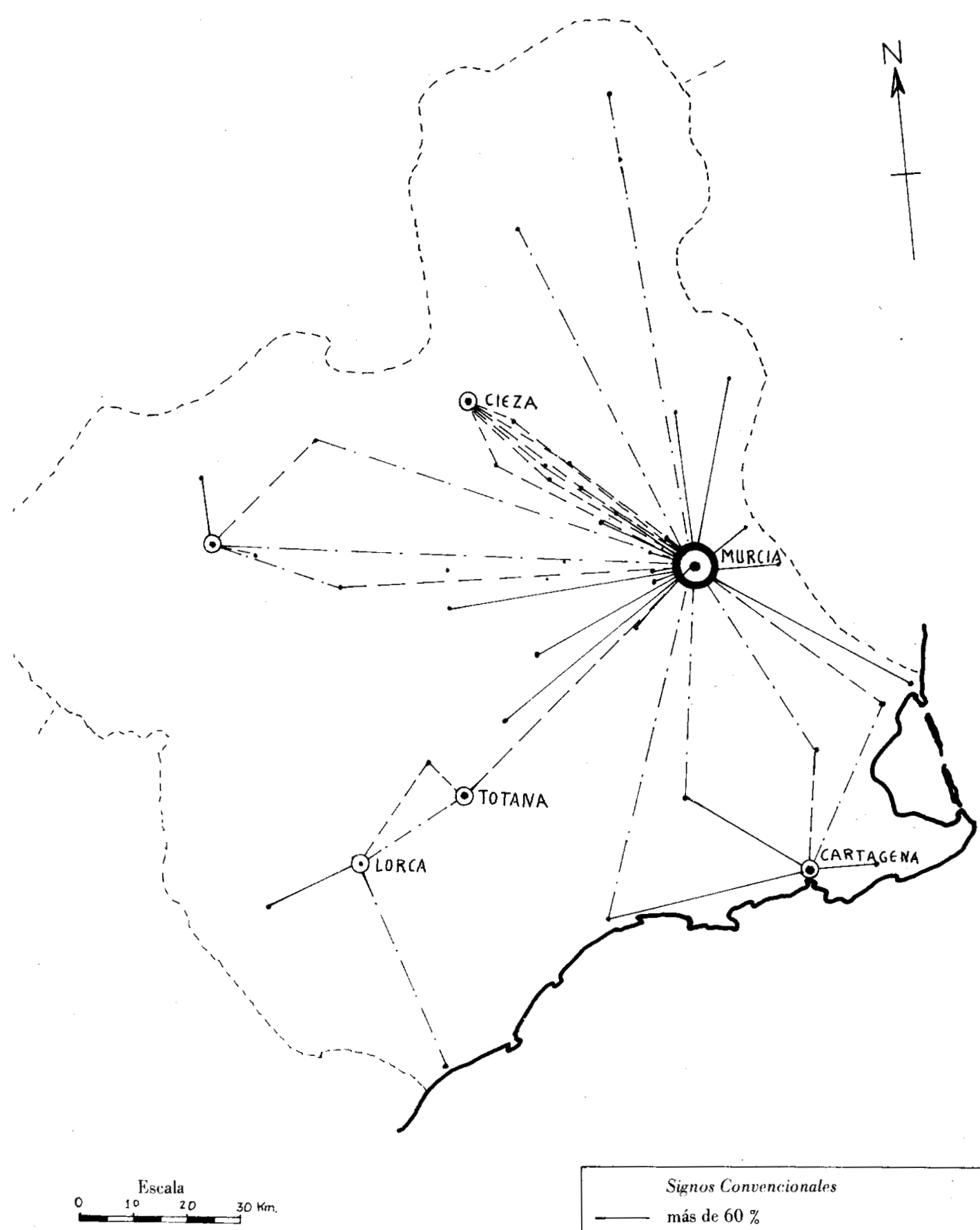

Signos Convencionales

más de $60 \%$

$\begin{array}{cl}- & \text { menos de } 30 \% \\ - & \text { entre } 30 \text { y } 60 \% \\ \text { L.C. activos }\end{array}$

Figura núm. 2 


\section{TOTAL DE UNIDADES FUNCIONALES}

Distribución de los Lugares Centrales

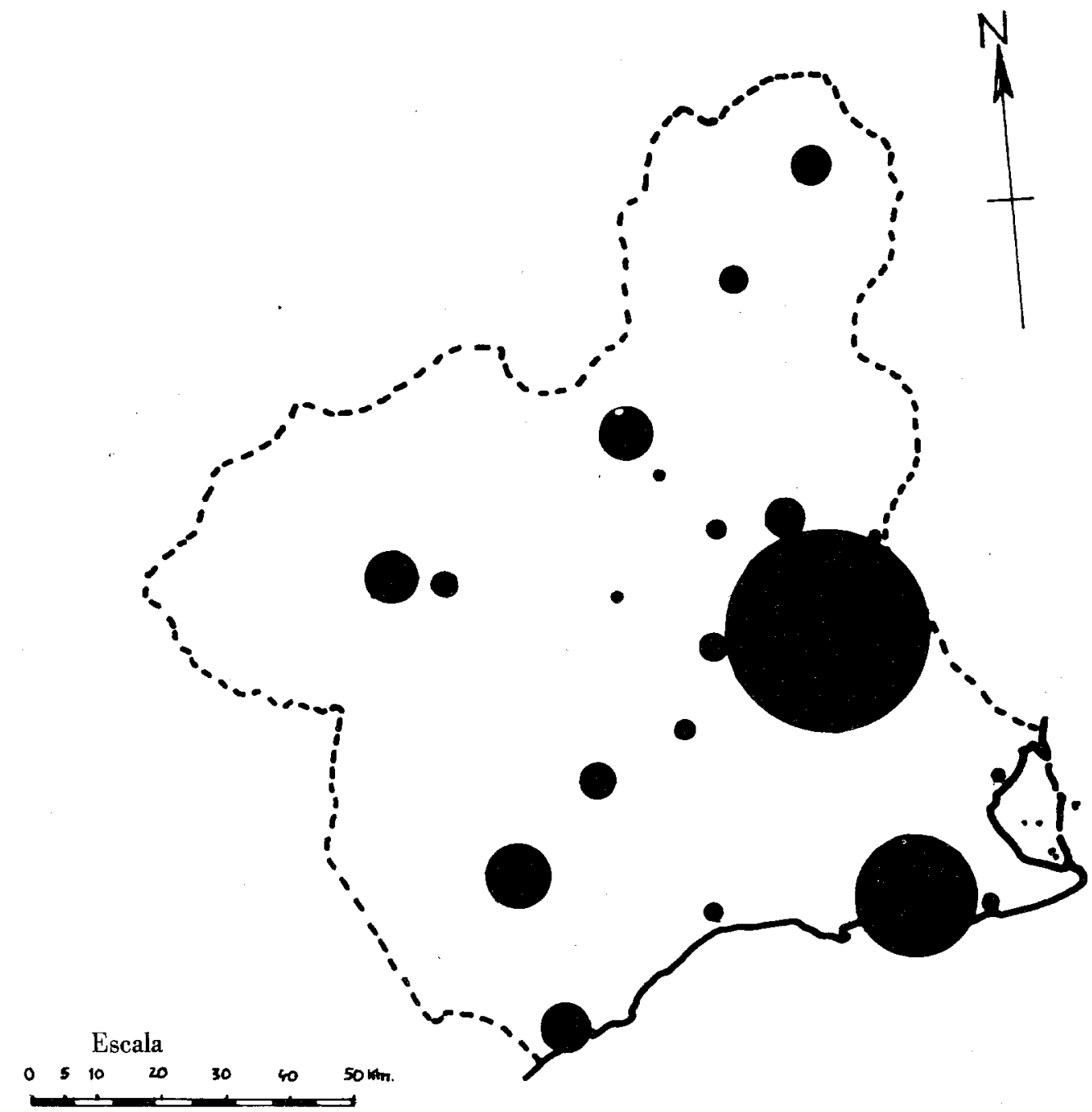

Figura núm. 3

Signos Convencionales

$$
\text { (r) } r=\sqrt{\frac{n}{2}}
$$

n número de Unidades Funcionales. 


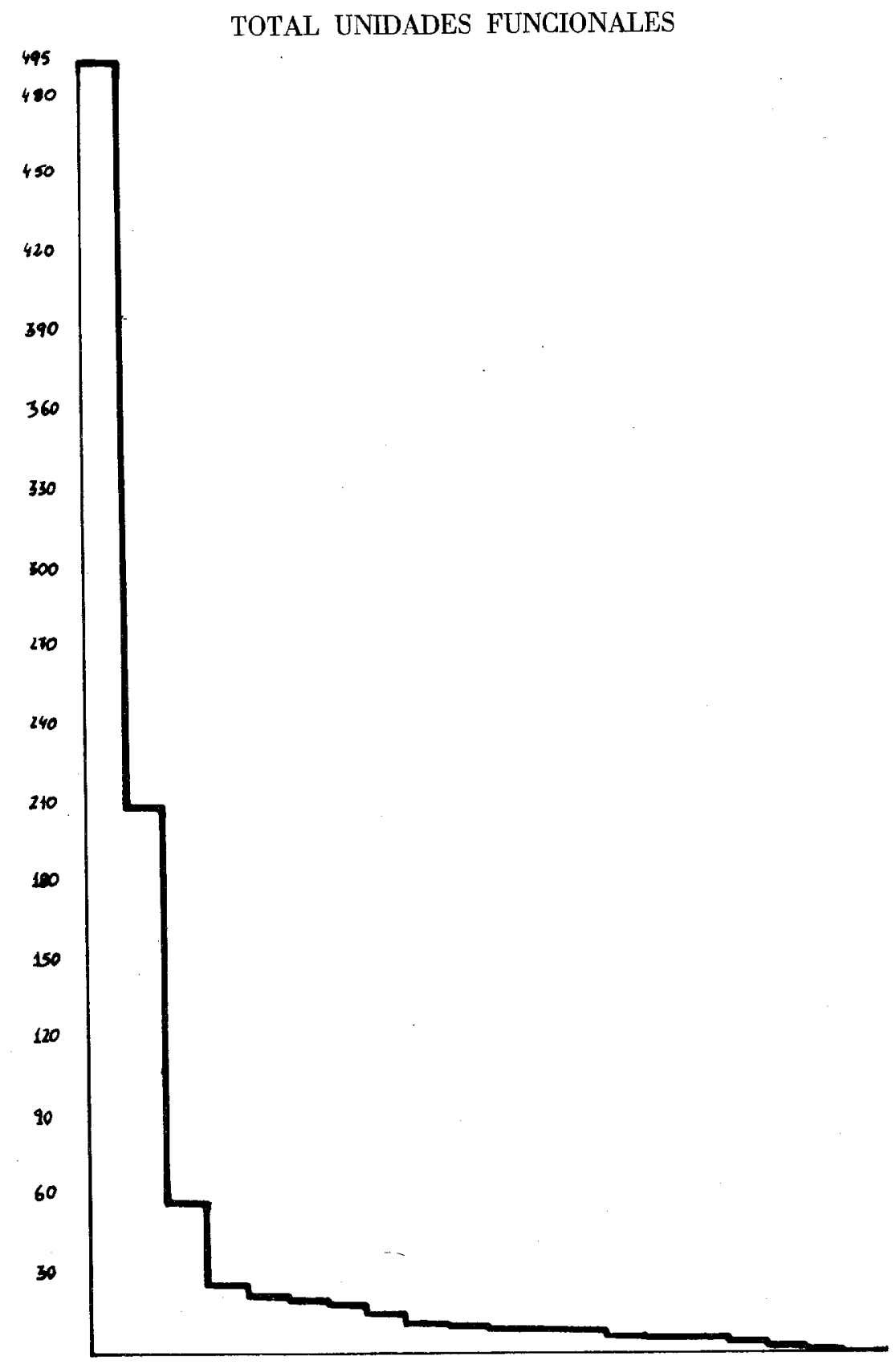

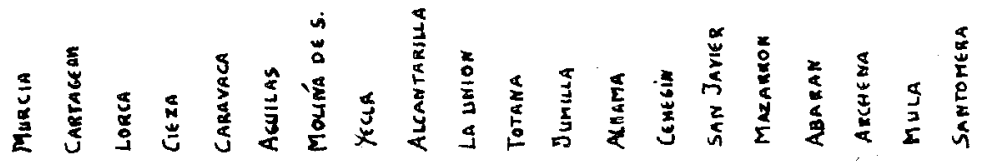

Figura núm. 4 
nes. De manera parecida a lo que sucede en el T.U.F., es a partir de Lorca (3. ${ }^{\circ}$ puesto en la jerarquía) donde desciende notablemente la variedad funcional, produciéndose un despegue muy fuerte en relación con los restantes L.C. que ven limitada su variedad funcional a cifras tan reducidas, que en el caso de los quince últimos puestos de la jerarquía, revisten un carácter prácticamente testimonial ya que sólo alcanzan una variedad funcional extendida a cuatro o cinco tipos de funciones.

Cabe advertir lo dicho antes, en el cuadro que sigue:

Cuadro número 2

\begin{tabular}{|c|c|c|}
\hline $\mathbf{A}$ & B & $\mathrm{C}$ \\
\hline 1 & Murcia . . . . . . . . & 44 \\
\hline 2 & 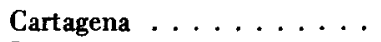 & 33 \\
\hline$\overline{3}$ & Lorca . . . . . . . . . . & 22 \\
\hline 4 & Cieza . . . . . . . . & 11 \\
\hline 5 & 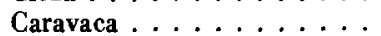 & 11 \\
\hline 6 & 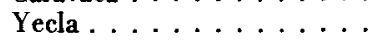 & 11 \\
\hline 7 & Aguilas . . . . . . . . . & 9 \\
\hline 8 & Alcantarilla ......... & 9 \\
\hline 9 & Molina de Segura . . . . . . & 8 \\
\hline 10 & 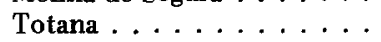 & 6 \\
\hline 11 & Jumilla . . . . . . . . . & $\mathbf{5}$ \\
\hline 12 & San Javier . . . . . . . & 5 \\
\hline 13 & La Unión. . . . . . . . . & 4 \\
\hline 14 & Alhama. . . . . . . . & 4 \\
\hline 15 & 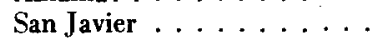 & 4 \\
\hline 16 & 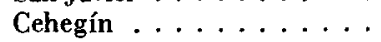 & 2 \\
\hline 17 & Abarán . . . . . . . . . & 2 \\
\hline 18 & Archena . . . . . . . & 2 \\
\hline 19 & Mula . . . . . . . . . & 1 \\
\hline 20 & Santomera . . . . . . . & 1 \\
\hline
\end{tabular}

A Número de orden en la jerarquía.

B Nombre de la ciudad.

C Variedad funcional.

De todo lo anterior se desprende que Murcia-ciudad sobresale ampliamente por su variedad funcional acumulada, resaltando sobre el conjunto de las demás. Cartagena y Lorca también mantienen una considerable variedad en su oferta, aunque más reducida que la capital.

Vienen a continuación Caravaca, Cieza y Yecla, centros comarcales ${ }^{9}$ de la Región con una importante gama de funciones especializadas con las cuales poder cumplir su cometido.

Sigue después Aguilas y Alcantarilla, con nueve funciones, y Molina de Segura con ocho. La razón de su dotación, quizás se deba a tratarse de ciudades con un crecido tamaño demográfico; la variedad funcional presente intenta cubrir las necesidades de esa población. En ciertos casos, como sucede en Aguilas, el anterior aspecto se ve agravado y condicionado por su especial distancia del principal centro abastecedor de la Región. blación.

Con seis funciones, aparece únicamente Totana; también núcleo importante de po-

9 En la Tesis Doctoral antes citada se define claramente la estructura de la jerarquía urbana de Murcia, en la cual Caravaca y Cieza desarrollan ese papel.

$$
-106 \text { - }
$$


En los restantes L.C. dotados, la variedad funcional resulta muy escasa, ampliada por el hecho de que en cada función el número de unidades es muy reducido: los establecimientos existentes son únicos dentro de su género.

Pueden comprobarse estadísticamente esos datos en la tabla número 2, ya citada, y en las figuras 5 y 6 donde se representan estos datos de forma gráfica.

\subsection{Indice de Centralidad}

Nos servimos de este índice por estar persuadidos de su gran utilidad ya que combina armónicamente tanto la V.F. y la distribución de las U.F. Estas a su vez dependen del rango concreto que cada bien posée.

Conocido el rango de los bienes, señalado al prịncipio, nos atenemos ahora a la fórmula conocida de Davies ${ }^{10}$.

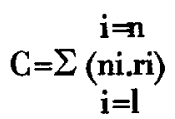

C Centralidad

ni Número de bienes existentes en $i$

ri Rango de los bienes arriba indicados

De acuerdo con ello el resultado es:

Cuadro número 3

Indice de Centralidad

\begin{tabular}{|c|c|c|c|}
\hline A & $\mathbf{B}$ & $\mathrm{C}$ & $\mathrm{D}$ \\
\hline 1 & Murcia & 405,88 & 55,81 \\
\hline 2 & Cartagena & 160,85 & 22,11 \\
\hline $\mathbf{3}$ & Lorea. . . & 41,90 & 5,79 \\
\hline 4 & Cieza . & 16,65 & 2,28 \\
\hline 5 & Caravaca & 14,39 & 1,97 \\
\hline 6 & Aguilas : & 13,50 & 1,85 \\
\hline 7 & Molina de Segura & 12,36 & 1,69 \\
\hline 8 & Yecla . . . . . . & 11,66 & 1,60 \\
\hline 9 & Alcantarilla & 8,51 & 1,17 \\
\hline 10 & La Unión. . & 6,99 & 0,96 \\
\hline 11 & Totana . & 5,83 & 0,80 \\
\hline 12 & Mazarrón. & 5,80 & 0,79 \\
\hline 13 & Jumilla . . & 4,87 & 0,66 \\
\hline 14 & Alhama. . . . . . . & 4,70 & 0,64 \\
\hline 15 & Cehegin $\ldots \ldots \ldots \ldots$ & 4,35 & 0,59 \\
\hline 16 & San Javier & 3,64 & 0,50 \\
\hline 17 & Abarán . . & 2,82 & 0,38 \\
\hline 18 & Archena & 1,29 & 0,17 \\
\hline 19 & Santomera . & 0,66 & 0,09 \\
\hline 20 & Mula $\ldots \ldots \ldots \ldots$ & 0,60 & 0,08 \\
\hline
\end{tabular}

A Número de orden en la jerarquía.

B Nombre de la ciudad.

C Indice de Centralidad.

D Porcentaje del anterior sobre el total.

10 DAVIES, W. D. K.: "Centrality and Central Place Hierarchy", en "Urban Studies", 1967, núm. 4, págs. 61.79 . 


\section{VARIEDAD FUNCIONAL}

Distribución en los Lugares Centrales

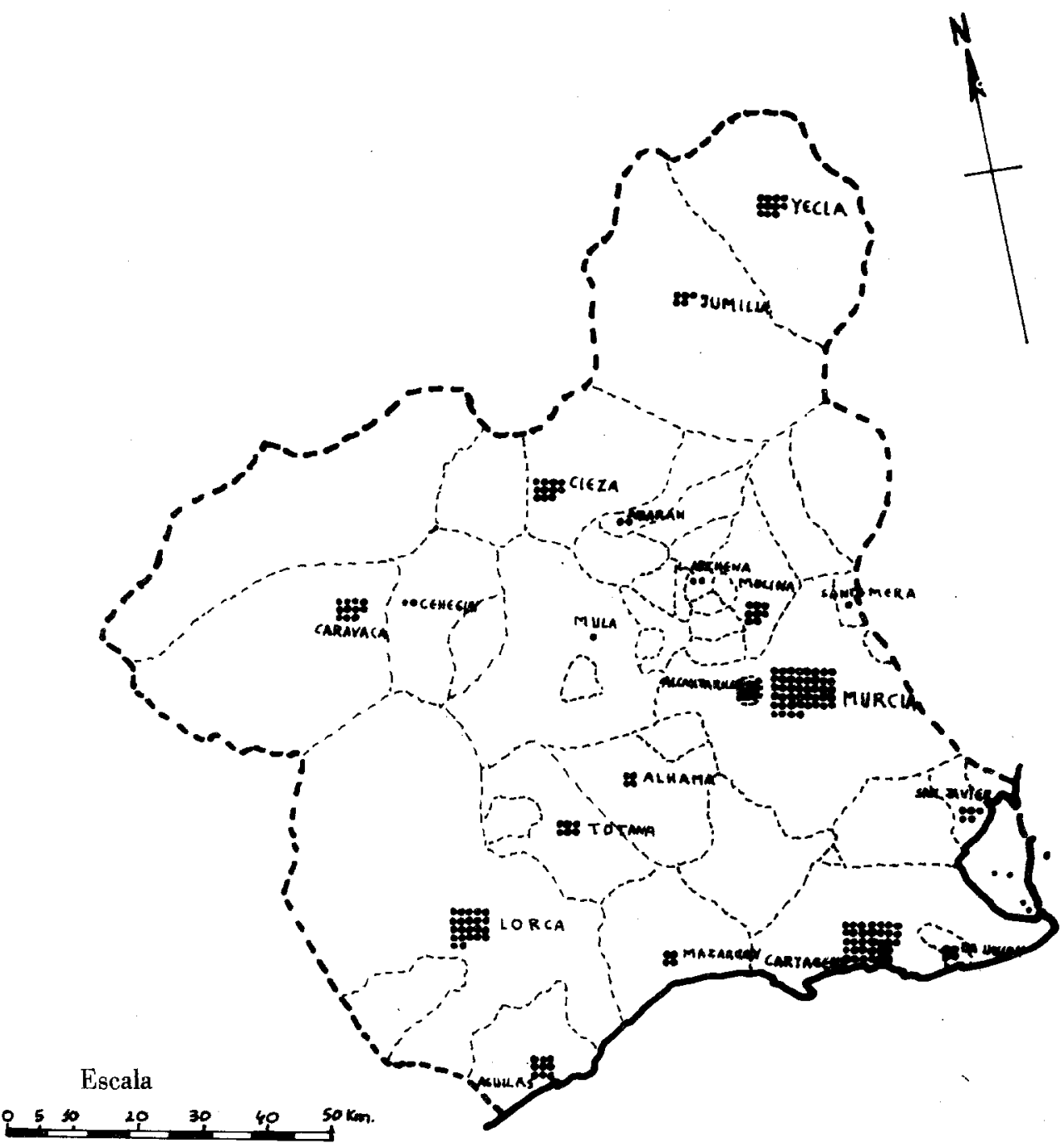

Signos Convencionales

- Una variedad funcional

Figura núm. 5 
VARIEDAD FUNCIONAL

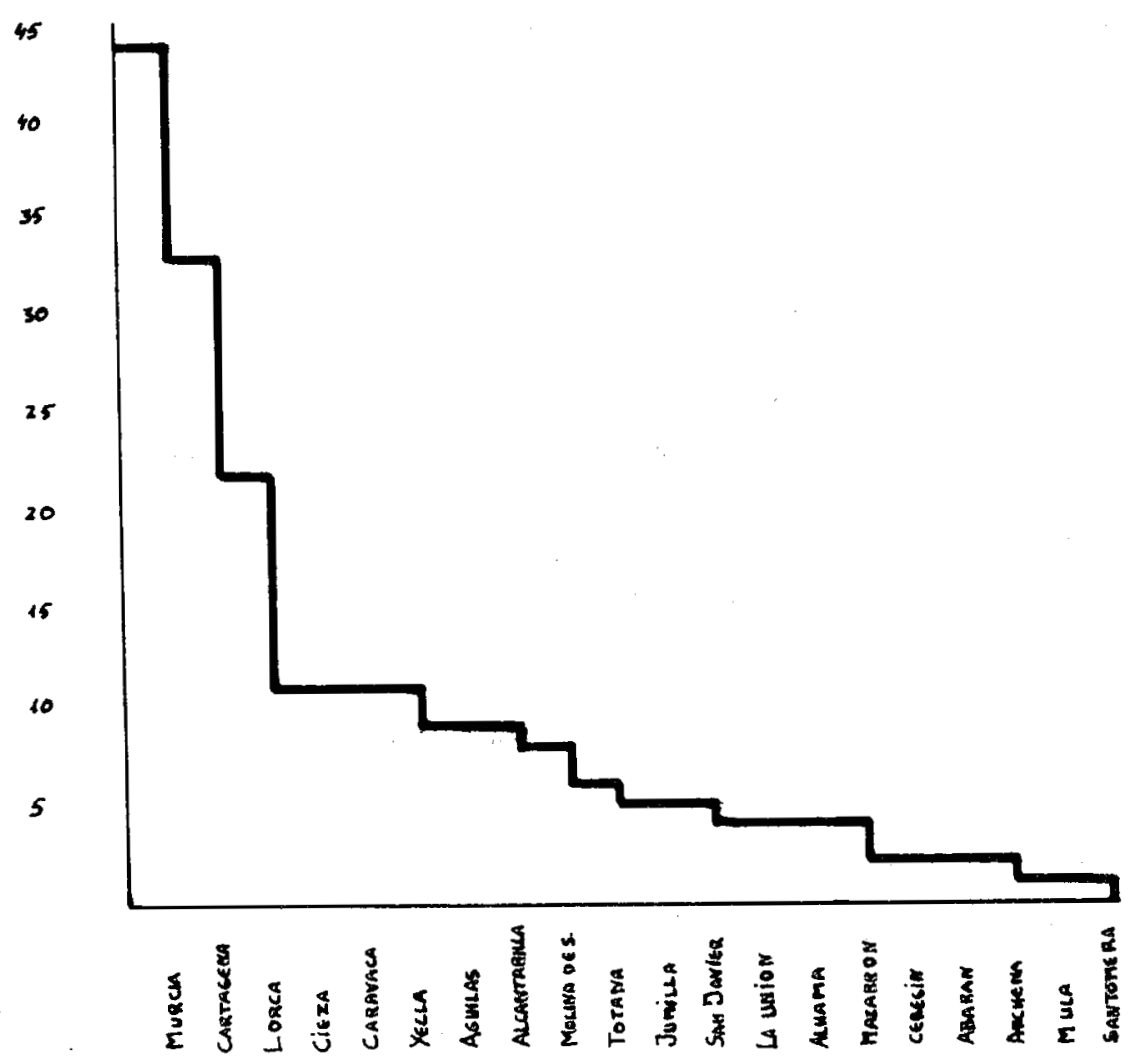

Figura núm. 6

$$
-109-
$$


INDICE DE CENTRALIDAD

Distribución en los Lugares Centrales

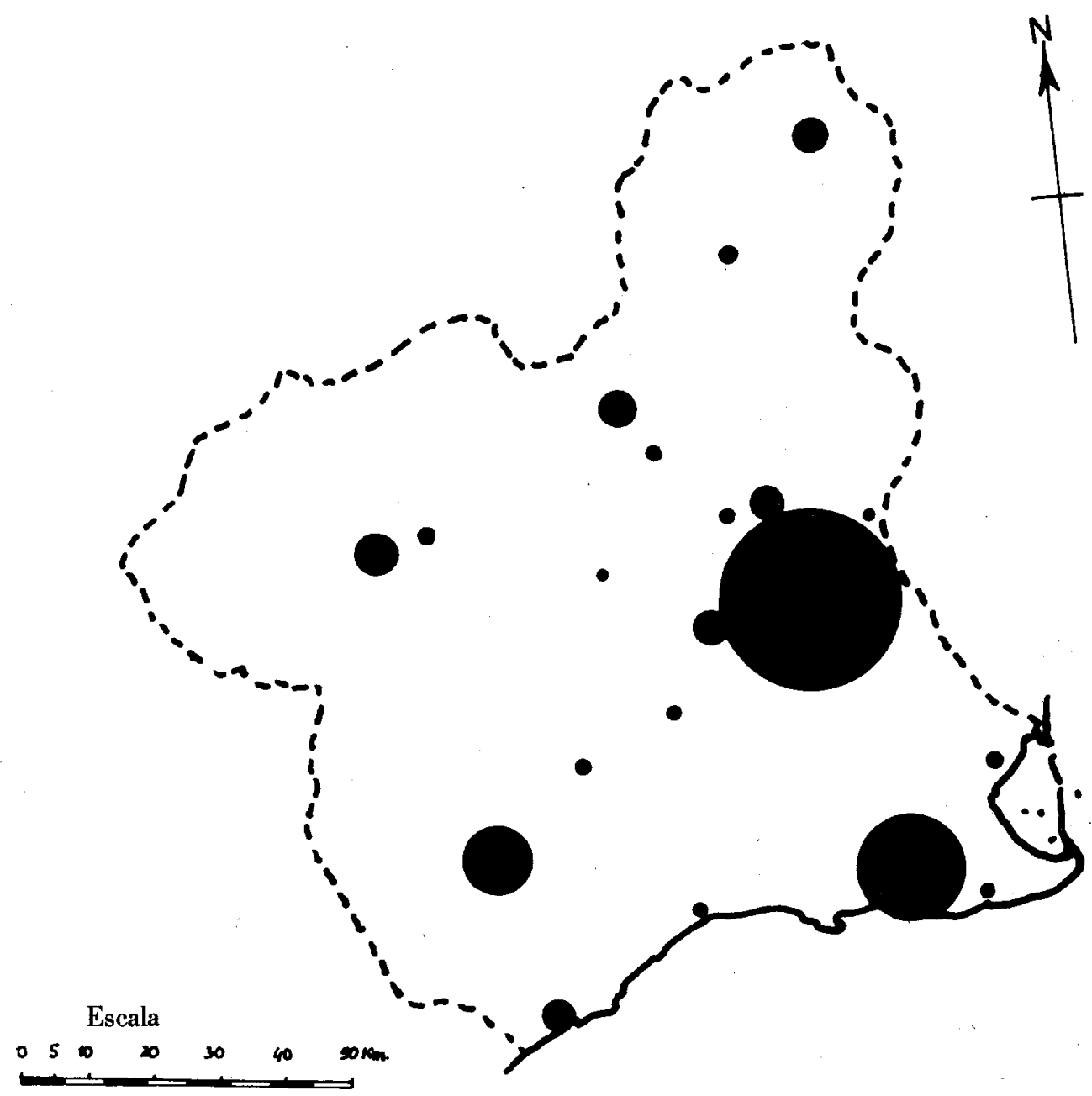

Signos Convencionales

$\longrightarrow r=\sqrt{\frac{n}{2}}$

n Indice de Centralidad

Figura núm. 7 
INDICE DE CENTRALIDAD

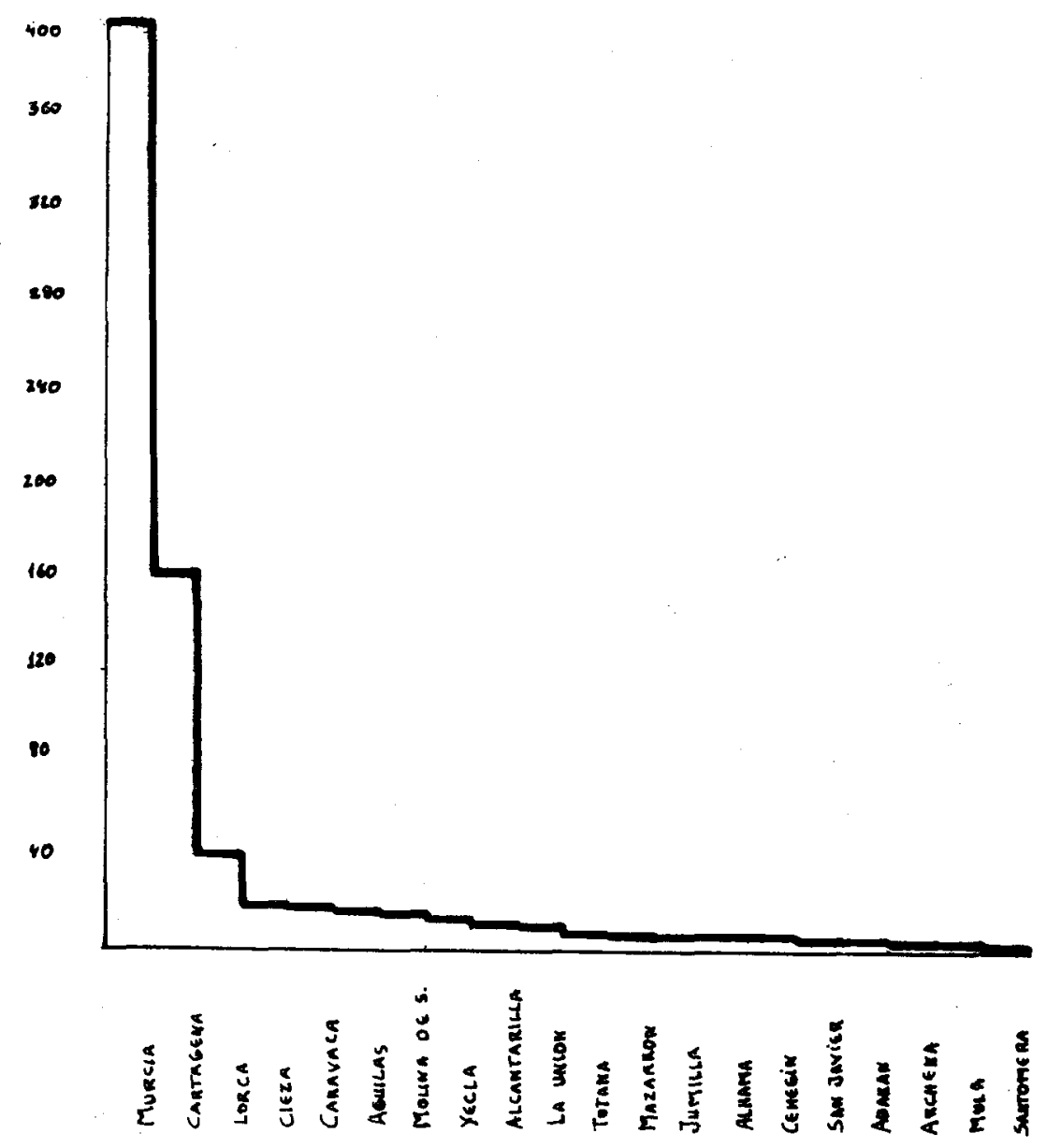

Figura núm. 8

$$
-111-
$$


El total de unidades siguiendo el I.C., asciende a 727,25 .

$\mathrm{Al}$ observar su reparto, de nuevo descuella la desigual distribución de los resultados entre los veinte L.C. equipados. La jerarquía resultante presenta a Murcia-ciudad con unos valores absolutos de 405,88 unidades, que contrastan con los de Cartagena que arroja un total de 160,85 . Supera a esta en más del doble de la magnitud alcanzada. En datos relativos los valores se elevan al $55,81 \%$ del total de la centralidad en el caso de Murcia y a 22,11 \% en Cartagena (figuras números 7 y 8 ).

Se produce después una variación fuerte en la relación jerárquica. Ello sucede en Lorca, ya que esta ciudad concentra un I.C. mucho más reducido que Cartagena y Murcia. Comparativamente significa tan sólo la cuarta parte del índice de Cartagena; y aproximadamente la décima del propio de Murcia. Queda pues, más cerca de los valores concentrados en los restantes L.C., si bien, todavía, su significación es más elevada que la de aquellos. Nos referimos a Cieza, Caravaca y Aguilas. Pero con relación a estos, resulta menor la centralidad acumulada por Lorca que la de esos lugares.

En los restantes, el I.C. va disminuyendo, con unos valores muy reducidos y similares entre sí. Sucede en Cieza y Caravaca, con 16,65 y 14,39; Aguilas, Molina de Segura y Yecla, con 13,50, 12,36 y 11,66 respectivamente. Sin embargo, todavía, en estas cinco unidades puede decirse que existe una cierta dotación funcional de los bienes estudiados. Ello demuestra un umbral de población suficiente que permite su subsistencia.

Continuamos con los lugares posteriores de la relación jerárquica, el valor del índice se va reduciendo hasta llegar a límites apenas significativos, y con un carácter casi simbólico. Esto sucede plenamente en lugares como Santomera y Mula, con valores inferiores a la unidad, o bien de manera menos acusada en Archena, Abarán, San Javier, Cehegín, Alhama y Jumilla (entre una y cinco unidades).

Los índices reducidos de centralidad de estas ciudades son poco significativos; tanto si nos atenemos a los datos absolutos como a los relativos.

\subsection{Comparación de las distintas jerarquías}

En este análisis del diverso grado de equipamiento funcional de los L.C., hemos confeccionado una tabla comparativa en que aparecen los L.C. clasificados según los cuatro criterios de referencia (cuadro 4).

Se pretende con ello confrontar el puesto ocupado por cada L.C., partiendo de la base de que en condiciones normales, y teóricamente perfectas, la jerarquía resultante, según el tamaño demográfico, marcaría la pauta en las otras relaciones. Es en esta donde ya se presentan las primeras anomalías dentro del marco regional, ya que no coinciden los 20 L.C. citados con los de mayor tamaño demográfico de la Región. Aparte de las alteraciones de orden que resultan; la no correspondencia se plantea en que existen ciertos lugares que quedan fuera de los aquí citados. Tal sucede con Torre Pacheco, Torres de Cotillas, Bullas, etc. Sin embargo otros de inferior tamaño demográfico quedân incluidos en ella como Mazarrón y Santomera.

\section{Centralidad-tamaño demográfico}

Resulta sin duda fundamental esta comparación, ya que la jerarquía producida a causa de la centralidad, es producto de combinar -como se expuso en su momento- la va- 


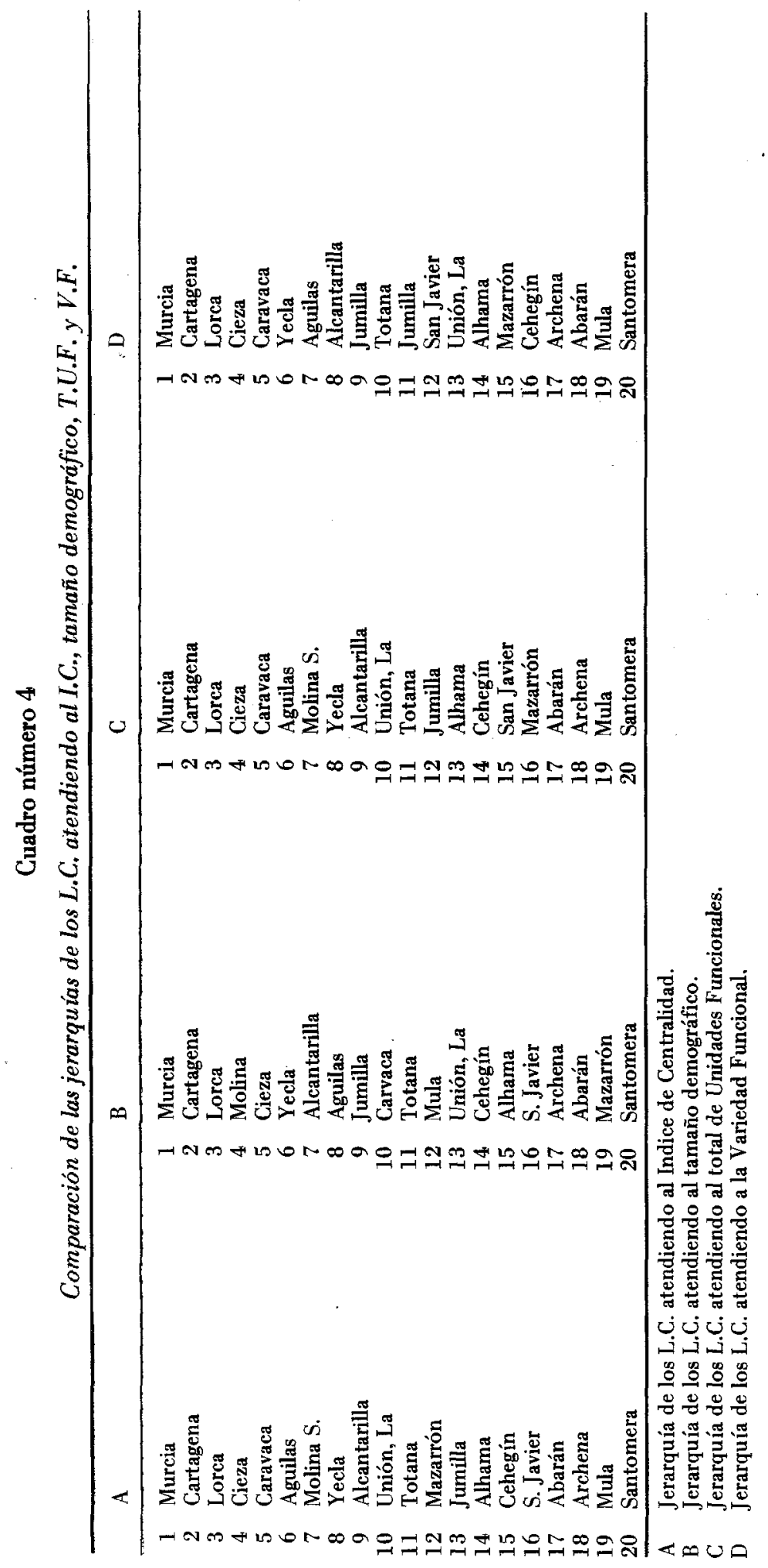


riedad funcional y la distribución del conjunto de unidades funcionales. Los resultados que se derivan son:

- Los tres primeros puestos de ambas relaciones mantienen una equivalencia, y lo mismo sucede con los situados en los lugares finales. En los puestos intermedios también hay equivalencia en el caso de San Javier y Totána. El resto se ve sometido a alteraciones.

- Incrementan su centralidad -en relación con el tamaño demográfico- Caravaca, Cieza, Aguilas, La Unión y Mazarrón, principalmente.

- Disminuyen en la comparación anterior, Molina de Segura, Yecla, Alcantarilla, entre los más notables.

\section{Tamaño demográfico-total unidades funcionales}

Las equivalencias en las dos jerarquías son escasas. Se reducen a las tres primeras ciudades de ambas: Murcia, Cartagena y Lorca.

Dos entre las que ocupan puestos intermedios, Totana y Cehegín y uno al final; Santomera. Predominan pues, ampliamente, las alteraciones producidas por infradotación de equipamiento como por supradotación. Es digno de resaltar en el caso primero la situación de Mula, Jumilla, Alcantarilla, Yecla y Molina de Segura. En el ámbito segundo, Caravaca, La Unión, Alhama, San Javier y Mazarrón. Destacamos de nuevo la gran cantidad de anomalías y las marcadas diferencias de estas en ambas relaciones jerárquicas. Como dato más significativo del conjunto, aparece la infradotación de Mula, y la supradotación de Caravaca.

\section{Total unidades funcionales-variedad funcional}

Son menores que en los otros casos las irregularidades existentes en estas jerarquías. Ascienden a siete. Ello sucede en los lugares que ocupan los cinco primeros puestos y los dos finales. Por otro lado los cambios que, como hemos visto, se producen en las ciudades que ocupan los rangos intermedios, no son de gran significación. En la mayoría de las alteraciones experimentadas se tratan sólo de tres o cuatro puestos. Quizás no es anormal esta realidad, ya que en buena lógica aquellos L.C. que poseen un equipamiento amplio en U.F. también lo tengan equivalente en la V.F., y viceversa. No obstante, las variaciones más señaladas se producen en: Infradotación en V.F.: Aguilas, Molina, La Unión, Alhama y Cehegín. Supradotación en V.F.: Yecla, San Javier, Mazarrón y Archena.

También cabría realizar una comparación atendiendo a la variedad funcional-centralidad y al total de unidades funcionales-centralidad. Renunciamos sin embargo a hacerlo ya que creemos poseen menos interés, dado que la centralidad es, como se sabe, el resultado directo de la suma de ambas magnitudes junto con los valores concretos de los rangos de cada bien.

\section{CONCLUSIONES}

Una vez finalizado nuestro análisis, creemos cabe obtener las siguientes conclusiones:

1. a ) La distribución de las U.F., V.F. e I.C. presentan unos valores muy distintos de unos lugares a otros. No hay equivalencia generalizada en los puestos que ocupan los L.C. en las diferentes jerarquías resultantes. Estas sólo se producen regularmente de for- 
ma repetida en aquellos que ocupan los primeros puestos, y en menor grado en los últimos. En todos los restantes son normales las alteraciones de orden.

$\left.2 .^{a}\right)$ Murcia-ciudad, resalta de una forma muy clara sobre el conjunto de todas las demás por los valores alcanzados; aquí la dotación, atendiendo a la V.F. (todas las funciones seleccionadas), al T.U.F. $(54,81 \%$ del total), es muy pronunciada. Supera con mucho a cualquiera otra ciudad de la Región. Estas magnitudes no ya sólo indican un principal puesto como capital administrativa y política de ella; también, como centro comercial, el más importante y especializado en estos bienes de rango elevado. Los bienes citados, se encuentran en unos valores proporcionales que superan ampliamente los que podrían corresponderle a su tamaño demográfico. La centralidad de Murcia en el comercio al por menor es muy importante. Su área funcional alcanza y supera los límites de la Provincia.

$\left.3 .^{2}\right)$ Cartagena también suponen en los diferentes elementos analizados un considerable grado de equipamiento, lo que demuestra sin paliativos su papel de segundo núcleo comercial de la Región. Pero los valores absolutos y relativos que alcanza son muy inferiores a los de Murcia-ciudad. De ahí que resulte muy alejada de la realidad una estructura bipolar en el sistema uibano regional.

$\left.4 .^{2}\right)$ Es también indiscutible la posición de tercer lugar que Lorca ocupa en todos los elementos estudiados, pero en comparación con los dos primeros L.C., su significación es mucho más reducida. Se aproxima en estos bienes, al resto de los L.C. de la relación. Unicamente atendiendo a la V.F. (22 funciones en su equipamiento) puede calificarse de notable su dotación. Sin embargo creemos no se debe infravalorar este aspecto, ya que por si solo nos indica la amplitud de la oferta de bienes selectos y la consiguiente existencia de áreas funcionales adecuadas que cubren los umbrales de población correspondientes. Quizás falte, en cierto modo, un mayor número de unidades funcionales, lo cual se explica por los reducidos efectivos demográficos.

$5 .^{\text {a) }}$ Después de estas ciudades se inicia con las restantes un descenso progresivo, hasta llegar a los puestos finales de las jerarquías, tanto en su dotación en T.U.F., V.F. como en I.C., no se aprecian saltos muy considerables entre ellas. Es cierto que si comparamos en términos absolutos los datos que ofrecen el primero y el último de cualquiera de las jerarquías, las diferencias son grandes en todos los aspectos. Pese a ello, es notoria la superior dotación funcional y de Centralidad de:

a) Cieza, Caravaca y Aguilas. Las dos primeras ya se han calificado claramente como los centros comarcales más notorios de la Región por servir a unas comarcas bien definidas y en una amplia gama de servicios.

b) Aguilas, aunque resulte con características similares en este aspecto a las anteriores, ha arrojado otros resultados equivalentes en el estudio de aspectos complementarios para poder incluirla en esa categoría. Sí concurren en ella circunstancias favorables (tamaño demográfico, lejanía a la capital, desarrollo turístico, etc.) que explican los resultados ofrecidos.

6. $\left.{ }^{2}\right)$ Molina de Segura, Yecla y Alcantarilla son las tres ciudades que siguen en la jerarquía atendiendo a la centralidad. Sin embargo Yecla, supera a las anteriores en V.F. Hecho que se explica por la considerable tradición histórica de cabecera comarcal del Altiplano (actualmente en regresión). No obstante su considerable distancia a Murciaciudad, el tipo concreto de poblamiento propio de su municipio y de los circundantes, favorecen la variedad del equipamiento. La centralidad de Molina de Segura y Alcantarilla se deben sobre todo al T.U.F. que poseen, derivadas del tamaño de su población.

7. ) A partir de estas ciudades se inicia un descenso más acusado. Sucede en La Unión, Totana o Mazarrón. Habrá que interpretarlo por la existencia de unidades fun- 
cionales destinadas meramente a cubrir las posibles necesidades de su población, sin que en ningún caso su umbral supere el área fúncional comprendida por su municipio. Es necesario tener presente que las ciudades antes citadas, junto con otras similares que puedan añadirse (Jumilla, Alhama o Cehegín) poseen un tamaño demográfico considerable, superior en todos los casos a los $12.000 \mathrm{~h}$. Población, que por sí sola, resulta suficiente como umbral de demanda para esos servicios.

8. ) Una situación similar es la que ofrece San Javier, Abarán, Archena, Mula y Santomera, aunque no cabe establecer una homologación simplista en las características que las asemejan, pues unas son antiguas cabeceras comarcales -hoy desaparecido ese papel- mientras que en otras, por el contrario, ha sido durante los últimos años cuando han conseguido su crecimiento más dinámico. 